serious AEs, discontinuations due to AEs, or clinically significant laboratory or ECG abnormalities were noted.

Figure: Mean (SE) percent change from baseline in SUA at steady state following verinurad $10 \mathrm{mg}$ or ALLO $300 \mathrm{mg}$ or in combination

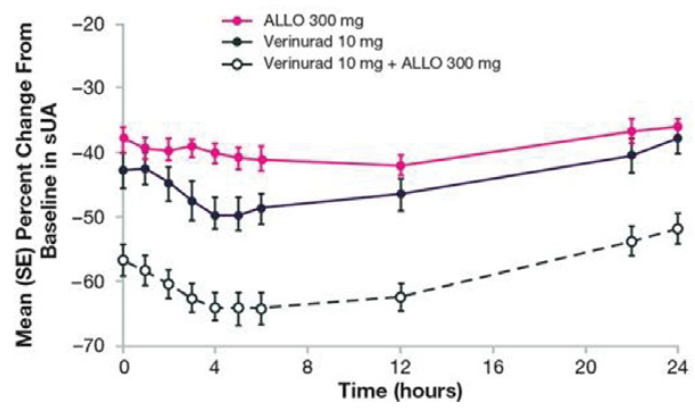

Conclusions: Although a modest drug-drug interaction was found between verinurad and ALLO, the combination was safe and well tolerated at the studied doses and resulted in greater reduction of sUA than either alone. These results support the evaluation of verinurad + ALLO as an alternative once-daily treatment option for hyperuricemia and gout.

Disclosure of Interest: J. Hall Employee of: Ardea Biosciences, Inc., M. Gillen Employee of: AstraZeneca, X. Yang Employee of: Ardea Biosciences, Inc., S. Liu Employee of: Ardea Biosciences, Inc., S. Walker Employee of: AstraZeneca, V. Clauson Employee of: Ardea Biosciences, Inc., M. Kankam: None declared DOI: 10.1136/annrheumdis-2017-eular.5308

\section{THU0433 PHARMACODYNAMIC AND PHARMACOKINETIC STUDY OF VERINURAD (RDEA3170) IN ADULT MALE SUBJECTS WITH MILD, MODERATE, AND SEVERE RENAL IMPAIRMENT: A PHASE 1, OPEN-LABEL STUDY}

W.B. Smith ${ }^{1}$, J. Hall ${ }^{2}$, J.K. Berg ${ }^{3}$, M. Kazimir ${ }^{3}$, A. Yamamoto ${ }^{2}$, S. Walker ${ }^{4}$, C. Lee ${ }^{2}$, T.C. Marbury ${ }^{5} .{ }^{1}$ Volunteer Research Group, Knoxville, TN; ${ }^{2}$ Ardea Biosciences, Inc., San Diego, CA; ${ }^{3}$ DaVita Clinical Research, Minneapolis, MN; ${ }^{4}$ Astrazeneca, Gaithersburg, MD; ${ }^{5}$ Orlando Clinical Research Center, Orlando, FL, United States

Background: Verinurad (RDEA3170) is a high-affinity, selective URAT1 inhibitor in development for the treatment of gout and asymptomatic hyperuricemia.

Objectives: This Phase 1, single-dose, open-label study investigated the pharmacodynamics (PD), pharmacokinetics (PK), and safety of oral verinurad in adult subjects with mild, moderate, or severe renal impairment and matched controls with normal renal function (NCT02219516).

Methods: Adult males aged 18-85 years were enrolled with a screening serum uric acid (sUA) 4.5-10 mg/dL and creatinine clearance calculated by CockcroftGault formula of 60 to $<90 \mathrm{~mL} / \mathrm{min}$ (mild renal impairment), 30 to $<60 \mathrm{~mL} / \mathrm{min}$ (moderate impairment), 15 to $<30 \mathrm{~mL} / \mathrm{min}$ (severe impairment), or $\geq 90 \mathrm{~mL} / \mathrm{min}$ (matched controls). Oral verinurad $15 \mathrm{mg}$ was administered once under fasted conditions. Serial blood and urine samples were taken $30 \mathrm{~min}$ before and up to 72 hours postdose. Safety assessments included laboratory, ECG, and vital sign parameters as well as adverse event (AEs) reporting.

Results: PD data were based on 7-8 subjects per group. Verinurad decreased sUA in all groups, with greatest changes in the normal function and mild renal impairment groups. Mean (SD) maximal \% change in sUA from baseline (Emax) was $-38.3(14.8) \%,-36.9(13.6) \%,-20.5(6.64) \%$, and $-12.6(6.94) \%$, respectively, in the normal function and mild, moderate, and severe renal impairment groups. Increase in the amount of excretion of uric acid due to verinurad treatment decreased in subjects with moderate and severe renal impairment. Plasma Cmax and AUC of verinurad increased with decreasing renal function. Verinurad at the $15 \mathrm{mg}$ dose was well tolerated, with no serious AEs, no subject withdrawals due to AEs, and no Renal Events Adjudication Committee (REAC)-adjudicated renal events during treatment. One patient in each renal impairment group had treatment-emergent AEs considered possibly related to verinurad, which were categorized as gastrointestinal in nature. There were no clinically meaningful changes noted in laboratory values or vital signs.

Conclusions: The sUA lowering effect of verinurad was observed across the spectrum of renal function. Consistent with the verinurad renal-dependent mechanism of action, decreasing sUA lowering was demonstrated with increasing renal impairment. Verinurad safety and tolerability were similar across all stages of renal impairment.

Disclosure of Interest: W. Smith Employee of: Volunteer Research Goup, J. Hall Employee of: Ardea Biosciences, Inc., J. Berg Employee of: DaVita Clinical Research, M. Kazimir Employee of: DaVita Clinical Research, A. Yamamoto Employee of: Ardea Biosciences, Inc., S. Walker Employee of: AstraZeneca, C. Lee Employee of: Ardea Biosciences, Inc., T. Marbury Employee of: Orlando Clinical Research Center

DOI: 10.1136/annrheumdis-2017-eular.5327

\section{THU0434 PHARMACODYNAMIC EFFECTS AND SAFETY OF VERINURAD (RDEA3170) IN COMBINATION WITH FEBUXOSTAT VERSUS FEBUXOSTAT ALONE IN ADULTS WITH GOUT: A PHASE 2A, OPEN-LABEL STUDY}

R. Fleischmann ${ }^{1}$, P. Winkle ${ }^{2}$, J. Hall $^{3}$, S. Valdez ${ }^{3}$, S. Liu ${ }^{3}$, X. Yan ${ }^{3}$, L. Hicks ${ }^{3}$, M. Hernandez-Illas ${ }^{4}$. ${ }^{1}$ University of Texas, Southwestern Medical Center, Metroplex Clinical Research Center, Dallas, TX; ${ }^{2}$ Anaheim Clinical Trials, Anaheim, CA; ${ }^{3}$ Ardea Biosciences, Inc., San Diego, CA; ${ }^{4}$ 4QPS MRA (Miami Clinical Research), Miami, FL, United States

Background: Verinurad (RDEA3170) is a high-affinity, selective URAT1 inhibitor in development for the treatment of gout and asymptomatic hyperuricemia. Objectives: This Phase $2 \mathrm{a}$, randomized, open-label, multicenter study investigated the multiple-dose pharmacodynamics (PD), pharmacokinetics (PK), and safety of oral verinurad in combination with febuxostat versus febuxostat alone in adults with gout (NCT02246673)

Methods: Patients aged $\geq 18$ and $\leq 75$ years with gout and serum uric acid (sUA) $>8 \mathrm{mg} / \mathrm{dL}$ were randomized to 1 of 5 cohorts to receive febuxostat (40 mg and $80 \mathrm{mg}$ ) alone and in combination with verinurad (dose range $2.5 \mathrm{mg}$ to $20 \mathrm{mg} ; 4$ treatment periods per cohort, each treatment period 7 days). Medications were administered once daily $\sim 30 \mathrm{~min}$ after breakfast. Colchicine $0.6 \mathrm{mg}$ for gout flare prophylaxis was initiated at approximately Day -14 (start of urate-lowering therapy [ULT]) washout) or Day -7 if not on ULT. Serial blood and urine samples were measured at preset intervals on Days -1, 1, 7, 14, 21, and 28 for PD and PK endpoints. Safety assessments included adverse events (AEs) and laboratory, electrocardiograms, and vital sign parameters.

Results: Sixty-four patients were randomized ( $n=12-14$ per cohort). Serum PD data pooled across cohorts demonstrated maximal \% decrease in sUA from baseline (Emax) at 8-12 $\mathrm{h}$ after dosing. Addition of verinurad to febuxostat decreased SUA in dose-dependent manner (Figure 1). Greater sUA reductions were observed for dose combinations of verinurad $\geq 5 \mathrm{mg}$ with febuxostat $40 \mathrm{mg}$ versus febuxostat $80 \mathrm{mg}$ alone. The rate of urinary uric acid excretion was reduced by febuxostat alone, but comparable to baseline levels with verinurad combined with febuxostat. Verinurad plasma exposures increased with verinurad dose and were comparable for febuxostat $40 \mathrm{mg}$ and $80 \mathrm{mg}$ doses. No drug-drug interaction on verinurad and febuxostat plasma PK parameters was observed. Verinurad at doses from $2.5 \mathrm{mg}$ to $20 \mathrm{mg}$ was well tolerated, with no serious AEs, withdrawals due to $A E s$, or renal-related events. The most frequent treatment-emergent $A E$ possibly related to study medication was pain in extremity, in 2 patients receiving verinurad. Laboratory values and vital signs showed no clinically meaningful changes. There were no cases of serum creatinine elevation $\geq 1.5 x$ baseline.

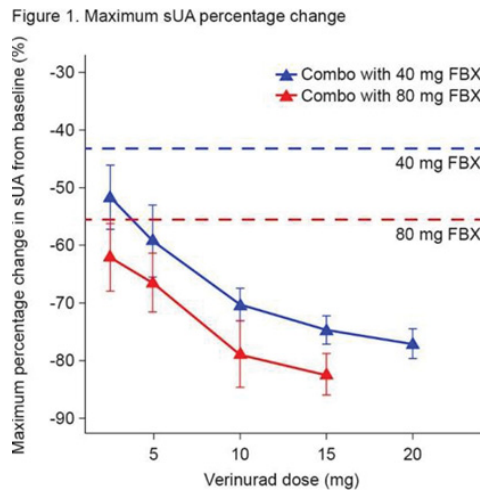

Conclusions: Verinurad coadministered with febuxostat dose-dependently decreased SUA while maintaining urine uric acid levels comparable to baseline. All dose combinations of verinurad and febuxostat in this study were generally well tolerated with no serious AEs or renal-related events during combination treatment.

Disclosure of Interest: R. Fleischmann Grant/research support from: Ardea Biosiences, Inc, P. Winkle Employee of: Anaheim Clinical Trials, J. Hall Employee of: Ardea Biosiences, Inc., S. Valdez Employee of: Ardea Biosiences, Inc., S. Liu Employee of: Ardea Biosiences, Inc., X. Yan Employee of: Ardea Biosiences, Inc., L. Hicks Employee of: Ardea Biosiences, Inc., M. Hernandez-Illas: None declared DOI: 10.1136/annrheumdis-2017-eular.5367

\section{THU0435 PHARMACODYNAMICS, PHARMACOKINETICS, AND SAFETY OF VERINURAD (RDEA3170) IN COMBINATION WITH FEBUXOSTAT VERSUS FEBUXOSTAT ALONE AND VERINURAD ALONE IN JAPANESE ADULTS WITH GOUT OR ASYMPTOMATIC HYPERURICEMIA: A PHASE 2A, OPEN-LABEL STUDY}

M. Shiramoto ${ }^{1}, \mathrm{~S}$. Liu ${ }^{2}$, Z. Shen ${ }^{2}$, J. Hall ${ }^{2} .{ }^{1}$ SOUSEIKAI PS Clinic, Fukuoka, Japan; ${ }^{2}$ Ardea Biosciences, Inc., San Diego, CA, United States

Background: Verinurad (RDEA3170) is a high-affinity URAT1 inhibitor in development for the treatment of gout and asymptomatic hyperuricemia. 